\title{
Predicting Factors for Allogeneic Blood Transfusion and Excessive Postoperative Blood Loss after Single Low-Dosage Intra-Articular Tranexamic Acid Application in Total Knee Replacement
}

\author{
Paphon Sa-ngasoongsong, Siwadol Wongsak, Noratep Kulachote, \\ Pongsthorn Chanplakorn, Patarawan Woratanarat, and Viroj Kawinwonggowit
}

Department of Orthopaedics, Faculty of Medicine, Ramathibodi Hospital, Mahidol University, Salaya, Thailand

Correspondence should be addressed to Siwadol Wongsak; siwadolrama@hotmail.com

Received 18 October 2016; Accepted 5 February 2017; Published 26 February 2017

Academic Editor: Giuseppe Filardo

Copyright (C) 2017 Paphon Sa-ngasoongsong et al. This is an open access article distributed under the Creative Commons Attribution License, which permits unrestricted use, distribution, and reproduction in any medium, provided the original work is properly cited.

\begin{abstract}
Background. Recently, intra-articular tranexamic acid (IA-TXA) application has become a popular method for perioperative blood loss (PBL) reduction in total knee replacement (TKR). Nevertheless, through our knowledge, no previous studies had shown the correlation perioperative factors and the risk of excessive PBL or need of blood transfusion (BT) after IA-TXA. Materials and Methods. A retrospective study was conducted in patients underwent 299 primary TKRs, using IA-TXA, during 2-year period (2013-2014). Patient's characteristic and perioperative data were reviewed and collected. PBL was measured as total hemoglobin loss (THL), estimated total blood loss (ETBL), and drainage volume per $\mathrm{kg}(\mathrm{DV} / \mathrm{kg})$. Excessive PBL was defined as PBL that exceeded 90th percentile. Results. From multivariate analysis, low preoperative hemoglobin ( $\mathrm{Hb}$ ) level and body mass index (BMI) were the significant predictors of postoperative BT ( $p<0.0001$ and 0.003 , resp.). Excessive THL significant associated with preoperative $\mathrm{Hb}$ $(p<0.0001)$. Excessive ETBL significantly associated with preoperative $\mathrm{Hb}$, height, preoperative range-of-motion, and creatinine clearance $(p<0.05$ all). Low BMI and large prosthesis size were the significant predictors of excessive DV/ $\mathrm{kg}(p=0.0001$ and 0.002 , resp.). Conclusions. Low preoperative $\mathrm{Hb}$ and BMI were the significant risks of postoperative transfusion after TKR with IA-TXA. Moreover, multiple perioperative factors could result in higher PBL.
\end{abstract}

\section{Background}

Intra-articular tranexamic acid (IA-TXA) application has recently become one of the most popular blood conservative methods in total knee replacement (TKR) due to its successfully proven effectiveness on reduction of postoperative blood loss (PBL) and blood transfusion (BT) without significant risk of postoperative complication [1-7]. Nonetheless, even with our best knowledge, of using this new technique, the amount of PBL is sometimes high and BT may be required, while these incidences seem to vary in different report studies, as $300-1,300 \mathrm{~mL}$ of PBL and $0 \%-20 \%$ of BT [8-13]. Although there have been numerous studies that showed predictors of blood loss and transfusion requirement after
TKR (such as age, gender, body mass index, preoperative hemoglobin $(\mathrm{Hb})$ level, use of postoperative anticoagulation, surgical time, and intraoperative blood loss $[14,15])$, the local application of tranexamic acid (TXA), which directly controls surgical bleeding, from raw surfaces and small noncoagulated blood vessels, might have different effects on these predicting factors of blood transfusion requirement presented in the previous literatures. Moreover, the risk factors associated with BT or PBL after using IA-TXA application have not yet been studied, and we thought that identifying patients with increased risk of considerable PBL and the need for perioperative BT is an important step toward establishing a better effective blood management strategy [16]. Therefore, this study aimed to identify the preoperative risk factors among 
the patients undergoing TKR with IA-TXA application which correlated with the substantial amount of postoperative blood loss and the need for postoperative blood transfusion. We hypothesized that some perioperative factors would influence the needs of postoperative transfusion and blood loss after using IA-TXA.

\section{Methods}

2.1. Study Design, Participants, and Inclusion and Exclusion Criteria. This was a singled-centered retrospective study and was approved by our institutional review board (protocol number 02-58-78). The electronic hospital database was used to identify the patients who underwent total joint replacement surgery from January 2013 to December 2014. The inclusion criteria were the patients who underwent primary total knee replacement, receiving operation from only one surgeon (VK) with the same surgical technique, prosthesis, and using IA-TXA application protocol the same as our previous study [17]. The exclusion criteria were patients who had simultaneous bilateral TKR or revision knee surgery, patients undertaking anticoagulant medication, and cases with acquired or congenital bleeding disorders.

\subsection{Surgical Procedure, IA-TXA Administration, and Periop-} erative Protocol. The surgical approach was medial parapatellar arthrotomy with midvastus approach. The anesthetic technique, either general or spinal anesthesia, was based on the decision of anesthesiologist, who was not involved in this study. The prosthesis was Nexgen ${ }^{\circledR}$ total knee system [Zimmer Inc., Warsaw, Indiana, USA] with patellar resurfacing in all patients, all prostheses were inserted with full cementation (Palacos, Heraeus Medical GmbH, Germany). A standard no. 8 drain tube was placed inside the knee joint and connected with high-pressure vacuum drain system (Drainobag ${ }^{\circledR}$ 600V Lock, B-Braun, Melsungen, Germany). The prepared IA-TXA solution, as $500 \mathrm{mg}$ of TXA with a total of $25 \mathrm{ml}$ volume, was then injected into the knee joint via drain tube after fascial and skin closure and application of compressive dressing. The drain was clamped for 2 hours before tourniquet deflation and then fully opened at ward. Postoperative care and transfusion guideline were the same as our previous study [17].

2.3. Data Collection and Outcome Measurement Method. The charts were reviewed, by using our electronic hospital database, to obtain required data including the patients' demographic data, perioperative data, and postoperative complications. Demographic data such as age, gender, weight, height, comorbid diseases and concurrent medications, preoperative knee osteoarthritis [OA] condition (as femorotibial angle measuring from standard long standing knee anteroposterior radiograph, knee osteoarthritis staging regarding Kellgren and Lawrence [KL] classification [18], and preoperative range-of-motion $[\mathrm{ROM}])$, American Society of Anesthesiologist (ASA) physical status, and preoperative laboratory values (hemoglobin [Hb], platelet count, activated partial prothrombin time [aPTT], international normalized ratio $[\mathrm{INR}]$, albumin and creatinine clearance $[\mathrm{CrCl}]$ ) were collected. Body mass index (BMI) and Charlson Comorbidity Index (CCI) were calculated. Perioperative and postoperative data, as side of operation, type of anesthesia, operative time, prosthesis size, intraoperative blood loss, drainage volume, hemoglobin on the 3rd postoperative day, amount of blood transfusion, and postoperative complications were recorded. Following the blood transfusion protocol from ASA guideline, transfusion was considered, when $\mathrm{Hb}$ was less than $8 \mathrm{gm} \%$ or the patient had positive anemic symptom (dyspnea, tachypnea, and hypoxemia) [19].

PBL was measured in 3 types: total hemoglobin loss (THL), estimated total blood loss (ETBL), and drainage volume per $\mathrm{kg}(\mathrm{DV} / \mathrm{kg})$. THL and ETBL were calculated using the specific formulae [20-22]. DV/kg was defined as total drainage volume divided by patient's weight in $\mathrm{kg}$. The definition of excessive PBL was by any type of PBL exceeding the 90th percentile cut-off point.

2.4. Statistical Analysis. Stata software version 11.0 (Stata Corp, College Station, Texas, USA) was used to analyze data. Continuous data and categorical data were presented as mean with standard deviation and number of cases with proportion. The association between each of the variables and blood transfusion or any types of excessive PBL was analyzed with univariate logistic regression analysis. Then the predicting factors with $p<0.15$ were entered into multivariate logistic regression analysis. Significant value was defined as $p<$ 0.05 .

\section{Results}

3.1. General (Characteristic and Correlation with Blood Loss). Between January 2013 and December 2014, a total of 251 patients with 299 primary unilateral total knee replacements (TKRs) (203 patients with unilateral TKRs and 48 patients with bilateral sequential TKRs), using intra-articular tranexamic acid (IA-TXA) application, were enrolled consecutively in this study. Among these patients, 208 of them were female $(83 \%)$, and the average patients' age \pm standard deviation was $69 \pm 8$ years. The incidence of blood transfusion was $5.4 \%$ (16 TKRs). Regarding the patients received transfusion, 15 of them $(94 \%)$ were transfused only 1 unit, while one case $(6 \%)$ needed 2 units of packed red cell transfusion. The mean THL, ETBL, and DV/kg was $1.6 \pm 0.9 \mathrm{~g} / \mathrm{dL}, 196 \pm 122 \mathrm{~mL}$, and $6.3 \pm$ $3.5 \mathrm{~mL} / \mathrm{kg}$, respectively. The 90th percentile THL, ETBL, and $\mathrm{DV} / \mathrm{kg}$ was $2.7 \mathrm{~g} / \mathrm{dL}, 348.6 \mathrm{~mL}$, and $10.6 \mathrm{~mL} / \mathrm{kg}$.

Table 1 demonstrated the characteristics of all 299 TKRs and the subgroup of patients based on the need of postoperative blood transfusion. There was a significant difference in preoperative factors, which were weight, BMI, preoperative $\mathrm{Hb}$, INR, serum albumin, and $\mathrm{CrCl}$ level, between blood transfusion (BT) group and non-BT group $(p<0.05$ all). Every postoperative blood loss (PBL) outcome in the BT group, including THL, ETBL, and DV/kg, was also significantly higher than the non-BT group ( $p<0.05$ all).

Table 2 showed the results of univariate logistic regression analysis for the effect of preoperative factors on BT and each type of excessive PBL. The significant preoperative risk factors 
TABLE 1: Characteristics of the 299 TKRs underwent primary TKR with IA-TXA application during 2013-2014 and risk factors for receiving blood transfusion.

\begin{tabular}{|c|c|c|c|c|}
\hline \multirow{2}{*}{ Patients characteristics } & \multirow{2}{*}{ Total $(n=299)$} & \multicolumn{2}{|c|}{ BT } & \multirow{2}{*}{$p$ value } \\
\hline & & Received BT $(n=16)$ & No BT $(n=283)$ & \\
\hline Age, year + & $69 \pm 8$ & $71 \pm 9$ & $69 \pm 8$ & 0.57 \\
\hline Male gender $\boldsymbol{\square}$ & $54(18)$ & $3(19)$ & $51(18)$ & 1.00 \\
\hline Right side $\mathbf{\square}$ & $145(48)$ & $10(63)$ & $135(48)$ & 0.31 \\
\hline Weight, kg + & $65 \pm 12$ & $59 \pm 13$ & $66 \pm 12$ & $0.04^{*}$ \\
\hline Height, $\mathrm{cm}+$ & $156 \pm 8$ & $157 \pm 8$ & $155 \pm 8$ & 0.48 \\
\hline $\mathrm{BMI}, \mathrm{kg} / \mathrm{m} 2+$ & $27.0 \pm 4.3$ & $24.0 \pm 4.4$ & $27.2 \pm 4.3$ & $0.007^{*}$ \\
\hline \multicolumn{5}{|l|}{ ASA grading $\mathbf{~}$} \\
\hline $1-2$ & $127(42)$ & $6(37)$ & $121(43)$ & 0.80 \\
\hline $3-4$ & $172(58)$ & $10(63)$ & $162(57)$ & \\
\hline $\mathrm{CCI}+$ & $3.7 \pm 1.0$ & $3.8 \pm 1.3$ & $3.7 \pm 1.0$ & 0.63 \\
\hline \multicolumn{5}{|l|}{ Comorbidities $\boldsymbol{\square}$} \\
\hline HT & $230(77)$ & $12(75)$ & $218(77)$ & 0.79 \\
\hline RA & $9(3)$ & $0(0)$ & $9(3)$ & 1.00 \\
\hline Antiplatelet agents usage $\boldsymbol{\square}$ & $35(12)$ & $2(13)$ & $33(12)$ & 1.00 \\
\hline OA KL grade 4 - & $197(67)$ & $12(75)$ & $185(65)$ & 0.59 \\
\hline Femorotibial angle, degree + & $-5.8 \pm 6.4$ & $-6.6 \pm 5.9$ & $-5.8 \pm 6.4$ & 0.65 \\
\hline Preoperative ROM, degree + & $109 \pm 19$ & $118 \pm 19$ & $109 \pm 19$ & 0.08 \\
\hline \multicolumn{5}{|l|}{ Preoperative laboratory values +} \\
\hline $\mathrm{Hb}, \mathrm{g} / \mathrm{dL}$ & $12.6 \pm 1.2$ & $11.1 \pm 1.3$ & $12.6 \pm 1.1$ & $<0.0001^{*}$ \\
\hline Platelet count, $\times 103 / \mathrm{mm} 3$ & $273 \pm 80$ & $256 \pm 68$ & $274 \pm 81$ & 0.40 \\
\hline aPTT, second & $27.0 \pm 2.4$ & $26.8 \pm 2.6$ & $27.1 \pm 2.4$ & 0.74 \\
\hline INR & $1.01 \pm 0.06$ & $1.05 \pm 0.10$ & $1.01 \pm 0.05$ & $0.02^{*}$ \\
\hline Albumin, $\mathrm{g} / \mathrm{dL}$ & $38.7 \pm 3.6$ & $36.8 \pm 5.3$ & $38.8 \pm 3.5$ & $0.05^{*}$ \\
\hline $\mathrm{CrCl}, \mathrm{ml} / \mathrm{min}$ & $70.6 \pm 23.4$ & $58.1 \pm 19.2$ & $71.3 \pm 23.4$ & $0.04^{*}$ \\
\hline General anesthesia & $16(5)$ & $1(6)$ & $15(5)$ & 0.59 \\
\hline Operative time, minute + & $80 \pm 17$ & $72 \pm 9$ & $81 \pm 17$ & 0.06 \\
\hline Large prosthesis size $\boldsymbol{\square}$ & $85(28)$ & $4(25)$ & $81(29)$ & 1.00 \\
\hline IBL, mL + & $47 \pm 35$ & $47 \pm 36$ & $44 \pm 15$ & 0.74 \\
\hline $\mathrm{IBL} / \mathrm{kg}, \mathrm{mL} / \mathrm{kg}+$ & $0.7 \pm 0.5$ & $0.7 \pm 0.5$ & $0.8 \pm 0.3$ & 0.67 \\
\hline $\mathrm{DV}, \mathrm{mL}+$ & $404 \pm 225$ & $550 \pm 320$ & $396 \pm 216$ & $0.01^{*}$ \\
\hline $\mathrm{DV} / \mathrm{kg}, \mathrm{mL} / \mathrm{kg}+$ & $6.3 \pm 3.5$ & $9.5 \pm 3.2$ & $6.1 \pm 3.3$ & $0.0005^{*}$ \\
\hline THL, g/dL + & $1.6 \pm 0.5$ & $2.3 \pm 1.5$ & $1.6 \pm 0.9$ & $0.008^{*}$ \\
\hline ETBL, mL + & $196 \pm 122$ & $495 \pm 136$ & $179 \pm 96$ & $<0.0001^{*}$ \\
\hline
\end{tabular}

BT: blood transfusion; +: value presented as mean \pm standard deviation; $\mathbf{~}$ : value presented as number of cases (percentage); BMI: body mass index; ASA: American Society of Anesthesiologists; CCI: Charlson Comorbidity Index; HT: hypertension; RA: rheumatoid arthritis; OA: osteoarthritis; KL: KellgrenLawrence; ROM: range-of-motion; Hb: hemoglobin; aPTT: activated partial thromboplastin time; INR: international normalized ratio; CrCl: creatinine clearance; IBL: intraoperative blood loss; IBL/kg: intraoperative blood loss per $\mathrm{kg}$; DV: drainage volume; DV/kg: drainage volume per kg; *: significant value with $p<0.05$.

for receiving $\mathrm{BT}$ and having excessive $\mathrm{PBL}$ from multivariate logistic regression analysis were illustrated in Table 3.

3.2. Risk Factors for Allogeneic Blood Transfusion. By univariate analysis, the preoperative factors that were significantly associated with BT were weight, BMI, preoperative ROM, $\mathrm{Hb}$, INR, creatinine clearance and albumin $(p<0.15$ all $)$ (Table 2). However, multivariate regression analysis demonstrated that only preoperative $\mathrm{Hb}$ and BMI were the significant predictors for receiving postoperative $\mathrm{BT}(\mathrm{Hb}$; odds ratio $[\mathrm{OR}]=0.23,95 \%$ confidence interval $[\mathrm{CI}]=0.12-0.43$, $p<0.0001$, and BMI; OR $=0.77,95 \% \mathrm{CI}=0.65-0.91, p=$ 0.003 ) (Table 3). The area under the curve (AUC) of this prediction model was $0.896(95 \% \mathrm{CI}=0.856-0.928)$.

\subsection{Risk Factors for Excessive PBL}

$T H L>2.7 \mathrm{~g} / \mathrm{dL}$. The significant preoperative factors, from univariate analysis, were preoperative $\mathrm{Hb}$ and platelet count ( $p \leq 0.0001$ and 0.118 , resp.) (Table 2). Multivariate analysis 


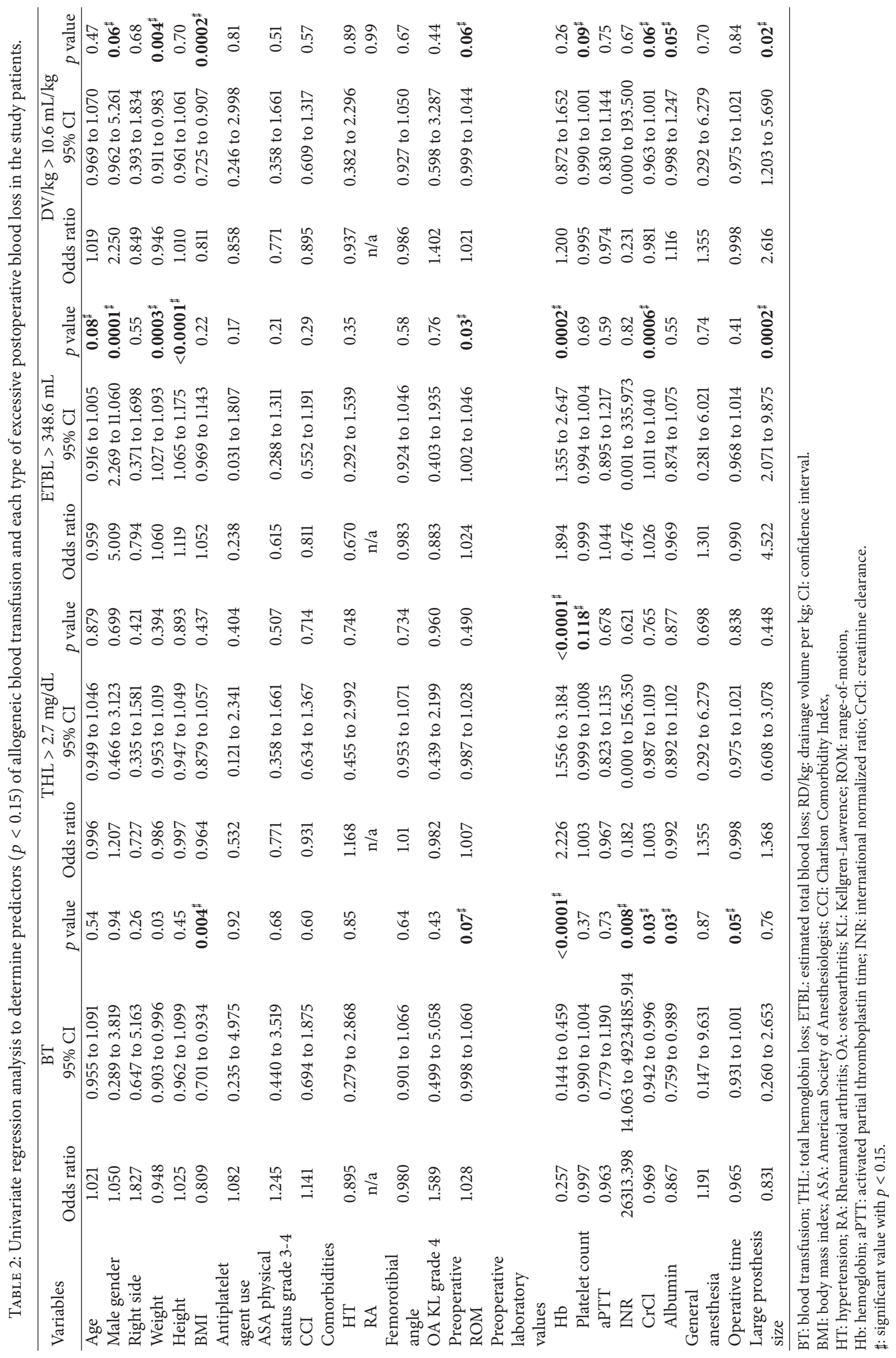


TABLE 3: Significant factors for allogeneic blood transfusion and excessive postoperative blood loss from multivariate logistic regression analysis.

\begin{tabular}{lccc}
\hline Significant factors & OR & $95 \%$ CI & $p$ value \\
\hline BT & & & \\
$\quad \mathrm{Hb}$ & 0.23 & 0.12 to 0.43 & $<0.0001$ \\
$\quad \mathrm{BMI}$ & 0.77 & 0.65 to 0.91 & 0.003 \\
$\mathrm{THL}>2.7 \mathrm{~g} / \mathrm{dL}$ & & & \\
$\quad \mathrm{Hb}$ & 2.23 & 1.56 to 3.18 & $<0.0001$ \\
$\mathrm{ETBL}>348.6 \mathrm{~mL}$ & & & \\
$\quad \mathrm{Height}$ & 1.11 & 1.05 to 1.17 & 0.0002 \\
$\mathrm{Hb}$ & 1.65 & 1.15 to 2.39 & 0.007 \\
$\quad$ Preoperative ROM & 1.03 & 1.00 to 1.06 & 0.02 \\
$\quad \mathrm{CrCl}$ & 1.02 & 1.01 to 1.04 & 0.006 \\
$\mathrm{DV} / \mathrm{kg}>10.6 \mathrm{~mL} / \mathrm{kg}$ & & & \\
$\quad \mathrm{BMI}$ & 0.78 & 0.69 to 0.88 & 0.0001 \\
$\quad$ Large prosthesis size & 3.91 & 1.67 to 9.14 & 0.002 \\
\hline
\end{tabular}

BT: blood transfusion; THL: total hemoglobin loss; ETBL: estimated total blood loss; DV/kg: drainage volume per $\mathrm{kg}$.

$\mathrm{Hb}$ : hemoglobin; BMI: body mass index; ROM: range-of-motion; $\mathrm{CrCl}$ : creatinine clearance.

indicated that only preoperative $\mathrm{Hb}$ was the independent risk factor $(\mathrm{OR}=2.23,95 \%$ CI 1.56-3.18, $p<0.0001)$ (Table 3). The AUC was 0.756 (95\% CI $=0.704-0.804)$.

$E T B L>348.6 \mathrm{~mL}$. The significant preoperative factors, from univariate analysis, were age, male gender, weight, height, preoperative $\mathrm{ROM}, \mathrm{Hb}$, creatinine clearance, and large prosthesis $(p<0.15$ all) (Table 2). Multivariate analysis demonstrated that the significant independent predictive factors were height $(\mathrm{OR}=1.11,95 \% \mathrm{CI} 1.05-1.17, p=0.0002)$, preoperative $\mathrm{Hb}(\mathrm{OR}=1.65,95 \% \mathrm{CI} 1.15-2.39, p=0.007)$, preoperative $\mathrm{ROM}(\mathrm{OR}=1.03,95 \% \mathrm{CI} 1.00-1.06, p=0.02)$, and $\mathrm{CrCl}(\mathrm{OR}=1.02,95 \% \mathrm{CI} 1.01-1.04, p=0.006)$ (Table 3$)$. The AUC was 0.804 (95\% CI 0.754-847).

$D V / \mathrm{kg}>10.6 \mathrm{~mL} / \mathrm{kg}$. The significant preoperative factors, from univariate analysis, were male gender, weight, BMI, preoperative $\mathrm{ROM}$, platelet count, $\mathrm{CrCl}$, albumin, and large prosthesis $(p<0.015$ all) (Table 2$)$. Multivariate analysis demonstrated that the significant independent predictive factors were BMI $(\mathrm{OR}=0.78,95 \%$ CI $0.69-0.88, p=0.0001)$ and large prosthesis $(\mathrm{OR}=3.91,95 \% \mathrm{CI} 1.67-9.14, p=0.002)$ (Table 3). The AUC was 0.782 (95\% CI 0.731-0.827).

\section{Discussion}

The result from this study showed that the significant independent risk factors for $\mathrm{BT}$ were lower preoperative $\mathrm{Hb}$ and lower BMI ( $p \leq 0.0001$ and 0.003 , resp.), which was consistent with the previously published reports on TKR without IA-TXA application [13-15, 23-27].

Regarding the risk factors for excessive PBL, the excessive THL $(>2.7 \mathrm{~g} / \mathrm{dL})$ was significantly associated with only higher preoperative $\mathrm{Hb}(p<0.0001)$, and the excessive ETBL $(>348.6 \mathrm{~mL})$ was significantly associated with the patients with greater height, preoperative $\mathrm{Hb}$, preoperative $\mathrm{ROM}$, and $\mathrm{CrCl}(p<0.05$ all), whereas excessive DV $/ \mathrm{kg}(>10.6 \mathrm{~mL} / \mathrm{kg})$ was significantly associated with lower BMI and larger prosthesis size ( $p=0.001$ and 0.002 , resp.).

The variation in significant predicting factors on each type of PBL in our study might be explained by the different methods of outcome measurement (THL, ETBL, and DV $/ \mathrm{kg}$ ) and their correlation on the effect of blood loss reduction from IA-TXA application. The significant association of higher preoperative $\mathrm{Hb}$ on excessive THL or ETBL could be explained by the effect of IA-TXA and drain clamping method on initial phase of blood loss. As a result from our previous studies, IA-TXA application with 2-hour drain clamping significantly reduced the drainage blood loss rate in the first 4 hours after clamp release [17]. The drainage volume during this period was the initial bleeding from surgical bed into the knee joint after tourniquet release which had the $\mathrm{Hb}$ concentration nearly the same as the patients' $\mathrm{Hb}$ and considered as the majority of postoperative THL. Therefore, the THL from patients with higher preoperative $\mathrm{Hb}$ would be greater than those with lower preoperative $\mathrm{Hb}$ considering that the blood volume loss from initial clot formation was nearly the same between individual subjects. Consequently, higher preoperative $\mathrm{Hb}$ would directly associate with the greater PBL outcomes which use the difference of $\mathrm{Hb}$ or $\mathrm{Hct}$ concentration, such as THL and ETBL.

The effect of height, preoperative $\mathrm{ROM}$, and $\mathrm{CrCl}$ on ETBL might be explained by the following reasons. The patients with greater height had higher estimated blood volume and greater ETBL would be a result from the specific formula [20-22]. Those with greater preoperative ROM would have lesser tightness of knee joint capsule and soft tissue, which resulted in larger knee volume after fascia closure. Therefore, the IA-TXA concentration in those with greater preoperative ROM would be lower than those with lesser preoperative ROM, which subsequently reduced the effect of blood loss reduction from this method. The effect of higher $\mathrm{CrCl}$ on ETBL might be explained by the better ability of TXA clearance in the patients' blood after systemic absorption [28] which resulted in lower serum TXA level and, therefore, decreased the effect of blood loss reduction, compared with the patients who had lower $\mathrm{CrCl}$. However, the effect of these factors on ETBL was still minimal, as $2 \%-11 \%$ higher in ETBL, compared with the effect of higher preoperative $\mathrm{Hb}$ on ETBL as shown in Table 3.

The effect of lower BMI and large prosthesis size on $\mathrm{DV} / \mathrm{kg}$ might be explained by the effect of the patients' weight on the outcome measurement and bleeding surface from bony cut in TKR. The patients with larger surface of bony cut from TKR (larger prosthesis size) resulted in greater bleeding surface from bony cut (greater DV/kg). Therefore, the patients with same size of bleeding surface and lower BMI would result in higher DV/kg than those who had higher BMI.

The strength of this study was that our protocol included only the same IA-TXA method with only one surgeon using the same surgical technique, prosthesis type, and postoperative care. Moreover, to our best knowledge, this was the first study that has demonstrated the correlation between blood 
loss and transfusion and the perioperative factors after using IA-TXA method. Based on our results, we recommended using the specific blood preservative strategy in those patients with significant risk for postoperative transfusion (low preoperative $\mathrm{Hb}$ and low $\mathrm{BMI}$ ), as the preoperative workup and treatment for anemia and malnutrition and/or the preoperative erythropoietin therapy $[16,29]$.

However, this study also had several limitations. First, this study was retrospective which might be inherently limited by the nature of the study and the completeness of patients' chart review. Secondly, our sample size was also relatively not large and might not be able to detect other significant predictive factors. Therefore, the multicentered prospective study with larger sample size should be performed to find out the other related factors. Lastly, although the IA-TXA application should be theoretically effective for reduction of blood loss and transfusion in TKR, this effect might not be the same, among the different regimens. Moreover, the application of IA-TXA method varied highly among the literature including the dosage, the application technique, and the use of drain clamp. Thus, the result of this study using a single low-dosage application together with 2-hour drain clamp might be different to the other methods.

\section{Conclusion}

This study showed that the patients undergoing primary TKR using IA-TXA application and having low preoperative $\mathrm{Hb}$ and BMI had a significant risk of postoperative transfusion. Moreover, multiple perioperative factors could result in higher PBL. Therefore, more specific strategy of blood loss preservation might be useful to address this group of patients.

\section{List of Abbreviations}

IA-TXA: Intra-articular tranexamic acid

PBL: Perioperative blood loss

TKR: Total knee replacement

BT: Blood transfusion

THL: Total hemoglobin loss

ETBL: Estimated total blood loss

$\mathrm{DV} / \mathrm{kg}$ : Drainage volume per $\mathrm{kg}$

Hb: Hemoglobin

BMI: Body mass index

TXA: Tranexamic acid

OA: Osteoarthritis

KL: $\quad$ Kellgren and Lawrence

ROM: Range-of-motion

ASA: American Society of Anesthesiologists

aPTT: Activated partial prothrombin time

INR: International normalized ratio

CrCl: Creatinine clearance

CCI: Charlson Comorbidity Index.

\section{Disclosure}

Paphon Sa-ngasoongsong and Pongsthorn Chanplakorn are orthopaedic surgeons who experienced and interested in intra-articular tranexamic acid injection methods with previous publication of this technique and work in Department of Orthopaedics, Faculty of Medicine, Ramathibodi Hospital, Mahidol University. Viroj Kawinwonggowit, Noratep Kulachote, and Siwadol Wongsak are experienced arthroplasty surgeon in Department of Orthopaedics, Faculty of Medicine, Ramathibodi Hospital, Mahidol University. Patarawan Woratanarat is an orthopaedic surgeon and expert in epidemiology who work in Department of Orthopaedics, Faculty of Medicine, Ramathibodi Hospital, Mahidol University.

\section{Competing Interests}

All of the authors declare that they have no conflict of interests.

\section{Authors' Contributions}

Paphon Sa-ngasoongsong, MD, is the main researcher who designed and performed study and prepared the manuscript. Siwadol Wongsak, MD, is an arthoplasty surgeon and corresponding author who assisted in data collection and manuscript preparation. Noratep Kulachote, MD, is an arthroplasty surgeon who helped in data collection. Pongsthorn Chanplakorn, MD, is an orthopaedic surgeon who helped in manuscript preparation. Patarawan Woratanarat, $\mathrm{MD}, \mathrm{PhD}$, is an orthopaedic surgeon and expert in epidemiology who advised the study design and methods. Viroj Kawinwonggowit, MD, is a senior arthroplasty surgeon who performed all of the operations.

\section{Acknowledgments}

The authors wish to thank Department of Orthopaedics, Faculty of Medicine, Ramathibodi hospital, Mahidol University, for all of the kind help and permission to carry out this study.

\section{References}

[1] S. Alshryda, M. Sukeik, P. Sarda, J. Blenkinsopp, F. S. Haddad, and J. M. Mason, "A systematic review and meta-analysis of the topical administration of tranexamic acid in total hip and knee replacement," Bone and Joint Journal, vol. 96, no. 8, pp. 10051015, 2014.

[2] C. Zhao-yu, G. Yan, C. Wei, L. Yuejv, and Z. Ying-ze, "Reduced blood loss after intra-articular tranexamic acid injection during total knee arthroplasty: a meta-analysis of the literature," Knee Surgery, Sports Traumatology, Arthroscopy, vol. 22, no. 12, pp. 3181-3190, 2014.

[3] M. Panteli, C. Papakostidis, Z. Dahabreh, and P. V. Giannoudis, "Topical tranexamic acid in total knee replacement: a systematic review and meta-analysis," Knee, vol. 20, no. 5, pp. 300-309, 2013.

[4] H. Shemshaki, S. M. A. Nourian, N. Nourian, M. Dehghani, M. Mokhtari, and F. Mazoochian, "One step closer to sparing total blood loss and transfusion rate in total knee arthroplasty: 
a meta-analysis of different methods of tranexamic acid administration," Archives of Orthopaedic and Trauma Surgery, vol. 135, no. 4, pp. 573-588, 2015.

[5] H. Wang, B. Shen, and Y. Zeng, "Comparison of topical versus intravenous tranexamic acid in primary total knee arthroplasty: a meta-analysis of randomized controlled and prospective cohort trials," Knee, vol. 21, no. 6, pp. 987-993, 2014.

[6] C. Yue, F. Pei, P. Yang, J. Xie, and P. Kang, "Effect of topical tranexamic acid in reducing bleeding and transfusions in TKA," Orthopedics, vol. 38, no. 5, pp. 315-324, 2015.

[7] Q. Wu, H.-A. Zhang, S.-L. Liu, T. Meng, X. Zhou, and P. Wang, "Is tranexamic acid clinically effective and safe to prevent blood loss in total knee arthroplasty? A meta-analysis of 34 randomized controlled trials," European Journal of Orthopaedic Surgery and Traumatology, vol. 25, no. 3, pp. 525-541, 2015.

[8] R. N. Maniar, G. Kumar, T. Singhi, R. M. Nayak, and P. R. Maniar, "Most effective regimen of tranexamic acid in knee arthroplasty: a prospective randomized controlled study in 240 patients knee," Clinical Orthopaedics and Related Research, vol. 470, no. 9, pp. 2605-2612, 2012.

[9] X. Aguilera, M. J. Martínez-Zapata, P. Hinarejos et al., “Topical and intravenous tranexamic acid reduce blood loss compared to routine hemostasis in total knee arthroplasty: a multicenter, randomized, controlled trial," Archives of Orthopaedic and Trauma Surgery, vol. 135, no. 7, pp. 1017-1025, 2015.

[10] J. G. Seo, Y. W. Moon, S. H. Park, S. M. Kim, and K. R. Ko, “The comparative efficacies of intra-articular and IV tranexamic acid for reducing blood loss during total knee arthroplasty," Knee Surgery, Sports Traumatology, Arthroscopy, vol. 21, no. 8, pp. 1869-1874, 2013.

[11] P. Sa-Ngasoongsong, T. Channoom, V. Kawinwonggowit et al., "Postoperative blood loss reduction in computer-assisted surgery total knee replacement by low dose intra-articular tranexamic acid injection together with 2-hour clamp drain: a prospective triple-blinded randomized controlled trial," Orthopedic Reviews, vol. 3, no. 2, article no. e12, 2011.

[12] J. Wong, A. Abrishami, H. El Beheiry et al., “Topical application of tranexamic acid reduces postoperative blood loss in total knee arthroplasty: a randomized, controlled trial," Journal of Bone and Joint Surgery_Series A, vol. 92, no. 15, pp. 2503-2513, 2010.

[13] I. Ahmed, J. K. K. Chan, P. Jenkins, I. Brenkel, and P. Walmsley, "Estimating the transfusion risk following total knee arthroplasty," Orthopedics, vol. 35, no. 10, pp. e1465-e1471, 2012.

[14] N. B. Frisch, N. M. Wessell, M. A. Charters, S. Yu, J. J. Jeffries, and C. D. Silverton, "Predictors and complications of blood transfusion in total hip and knee arthroplasty," Journal of Arthroplasty, vol. 29, no. 9, pp. 189-192, 2014.

[15] S. C. Sizer, J. J. Cherian, R. D. K. Elmallah, T. P. Pierce, W. B. Beaver, and M. A. Mont, "Predicting blood loss in total knee and hip arthroplasty," Orthopedic Clinics of North America, vol. 46, no. 4, pp. 445-459, 2015.

[16] M. E. Keating and J. B. Meding, "Perioperative blood management practices in elective orthopaedic surgery," Journal of the American Academy of Orthopaedic Surgeons, vol. 10, no. 6, pp. 393-400, 2002.

[17] P. Sa-Ngasoongsong, S. Wongsak, P. Chanplakorn et al., "Efficacy of low-dose intra-articular tranexamic acid in total knee replacement; a prospective triple-blinded randomized controlled trial," BMC Musculoskeletal Disorders, vol. 14, article 340, 2013.
[18] J. H. Kellgren and J. S. Lawrence, "Radiological assessment of osteo-arthrosis," Annals of the Rheumatic Diseases, vol. 16, no. 4, pp. 494-502, 1957.

[19] "Practice guidelines for perioperative blood transfusion and adjuvant therapies: an updated report by the American Society of Anesthesiologists Task Force on Perioperative Blood Transfusion and Adjuvant Therapies," Anesthesiology, vol. 105, no. 1, pp. 198-208, 2006.

[20] P. Seeber and A. Shander, Basics of Blood Management, Blackwell Publishing, Malden, Mass, USA, 1st edition, 2007.

[21] M. E. Brecher, T. Monk, and L. T. Goodnough, "A standardized method for calculating blood loss," Transfusion, vol. 37, no. 10, pp. 1070-1074, 1997.

[22] J. B. Gross, "Estimating allowable blood loss: corrected for dilution," Anesthesiology, vol. 58, no. 3, pp. 277-280, 1983.

[23] B. E. Bierbaum, C. Hill, J. J. Callaghan et al., "An analysis of blood management in patients having a total hip or knee arthroplasty," Journal of Bone and Joint Surgery - Series A, vol. 81, no. 1, pp. 2-10, 1999.

[24] F. Mesa-Ramos, M. Mesa-Ramos, C. Maquieira-Canosa, and P. Carpintero, "Predictors for blood transfusion following total knee arthroplasty: A Prospective Randomised Study," Acta Orthopaedica Belgica, vol. 74, no. 1, pp. 83-89, 2008.

[25] S. Guerin, C. Collins, H. Kapoor, I. McClean, and D. Collins, "Blood transfusion requirement prediction in patients undergoing primary total hip and knee arthroplasty," Transfusion Medicine, vol. 17, no. 1, pp. 37-43, 2007.

[26] H. Yoshihara and D. Yoneoka, "Predictors of allogeneic blood transfusion in total hip and knee arthroplasty in the United States, 2000-2009," Journal of Arthroplasty, vol. 29, no. 9, pp. 1736-1740, 2014.

[27] M. R. Bong, V. Patel, E. Chang, P. S. Issack, R. Hebert, and P. E. Di Cesare, "Risks associated with blood transfusion after total knee arthroplasty," Journal of Arthroplasty, vol. 19, no. 3, pp. 281287, 2004.

[28] P. Sa-Ngasoongsong, P. Chanplakorn, S. Wongsak et al., "An in vivo study of low-dose intra-articular tranexamic acid application with prolonged clamping drain method in total knee replacement: clinical efficacy and safety," BioMed Research International, vol. 2015, Article ID 164206, 6 pages, 2015.

[29] T. P. Sculco, A. Baldini, and E. M. Keating, "Blood management in total joint arthroplasty," Instructional Course Lectures, vol. 54, pp. 51-66, 2005. 


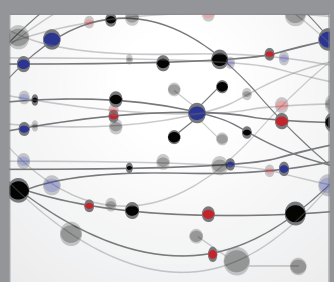

The Scientific World Journal
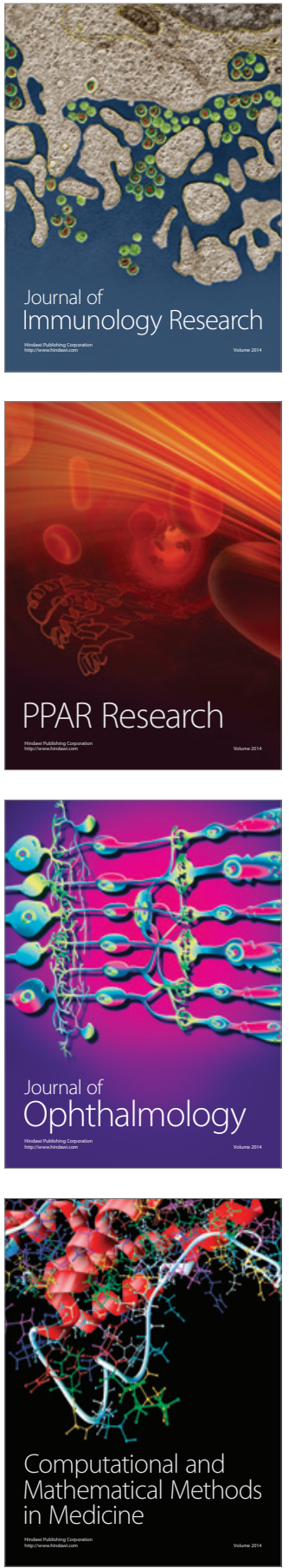

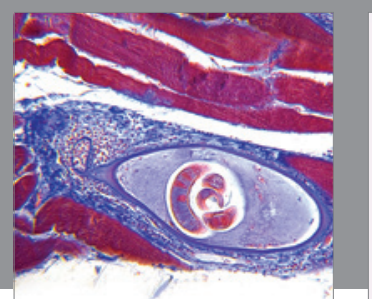

Gastroenterology Research and Practice
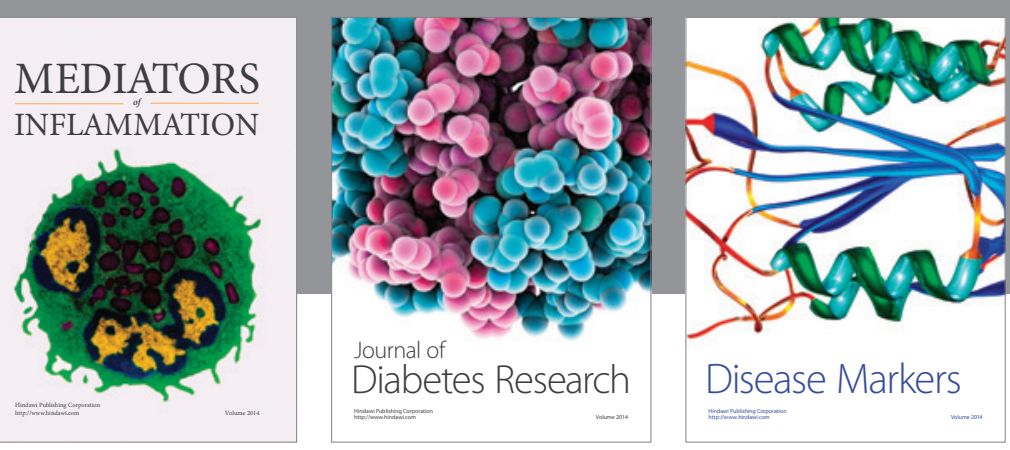

Disease Markers

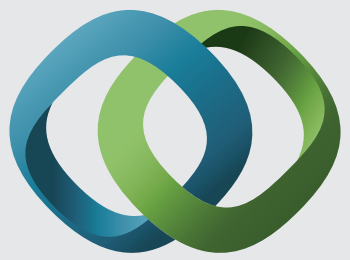

\section{Hindawi}

Submit your manuscripts at

https://www.hindawi.com
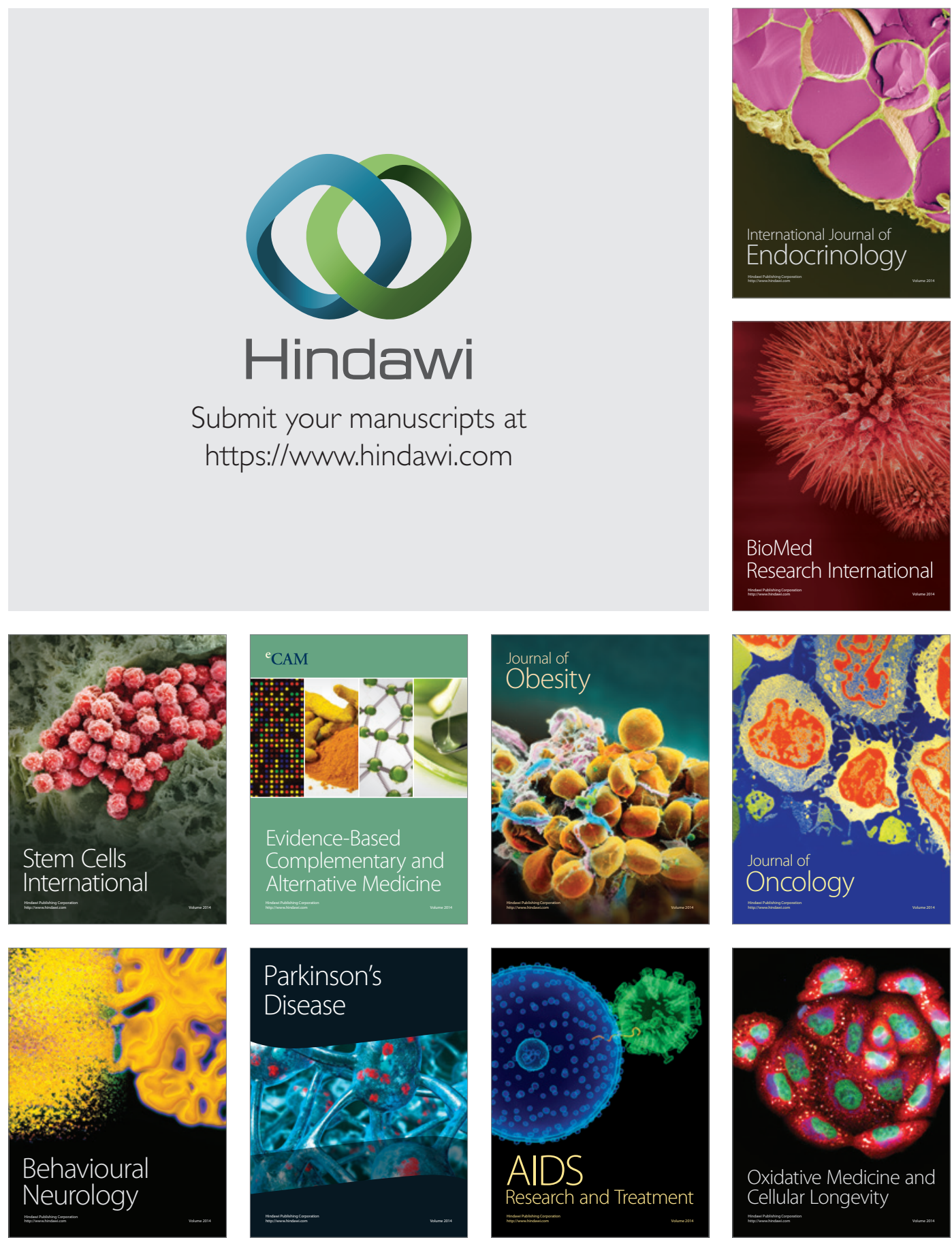